\title{
Modification de l'encrassement de surfaces d'échange de chaleur par le lait, le lactosérum et des liquides modèles sous l'action d'un champ électrique
}

\author{
F.-L. KERHERVÉ *, J.P. LABBÉ **, A. QUEMERAIS ***, \\ J.-C. REGGIANI ****, J. PAGETTI ****, G. DAUFIN * \\ * INRA, Laboratoire de Recherches de Technologie laitière \\ 65, rue de Saint-Brieuc, 35042 Rennes Cedex, France \\ ** Ecole Nationale Supérieure de Chimie, Laboratoire de Corrosion \\ 11, rue Pierre-et-Marie-Curie, 75231 Paris Cedex 05, France \\ *** Université de Rennes I, Faculté des Sciences, \\ Laboratoire de Spectroscopie du Solide, U.A. CNRS 1202 \\ Avenue du Général-Leclerc, Campus de Beaulieu, 35042 Rennes Cedex, France \\ **** Laboratoire de Corrosion et Traitements de Surfaces, \\ Faculté des Sciences et Techniques de Besançon \\ 32, rue Mégevand, 25030 Besançon Cedex, France
}

\section{Résumé}

L'application d'un champ électrıque à une surface d'échange de chaleur en modifie considérablement l'encrassement, les modifications dépendant à la fois de la polarisation électrique et de la nature des fluides. D'une manière générale, les fluides contenant protéines, phosphates et calcium donnent, sous polarisation (cathodique ou anodique) des masses de dépôts plus élevées qu'en encrassement libre. Les fluides contenant uniquement du phosphate et du calcium donnent des dépôts plus importants en cathodique et nettement moins importants en anodique, comparés à ceux obtenus en encrassement libre. Ces résultats, peu encourageants pour la lutte contre l'encrassement, s'expliquent essentiellement par l'accélération de la précipitation des phosphates en cathodique et de celle des protéines en anodique.

L'analyse des courbes transitoires tracées dans le domaine cathodique permet d'obtenir des informations recoupant celles recueillies par l'analyse quantitative et qualitative du dépôt telles que le recouvrement de la surface par ce dépôt, son adhérence et sa qualité isolante. Il pourrait s'agir là d'un principe intéressant pour un capteur d'encrassement, moyennant des travaux complémentaires (étalonnage, ....).

Enfin, du point de vue du mécanisme même de l'encrassement, les résultats montrent une interaction entre les phosphates, les ions calcium et les protéines. Les complexes peu solubles formés confirment le rôle essentiel des ions phosphates sur le niveau même de l'encrassement.

Mots clés: Encrassement - Surface d'échange de chaleur - Champ électrique - Lait Lactosérum - Spectrophotométrie de photoélectrons - Spectrophotométrie infrarouge. 


\section{Summary}

Modification of heat transfer surface fouling by milk, whey and model fluids under an electric field.

The effect of an electric field on titanium fouling was studied by using a heat exchange model equipped with electrodes and a standard electrical assembly. Model solutions were used as well as more complex fluids (milk, whey), preheated at $72{ }^{\circ} \mathrm{C}$.

Applying an electric field results in a considerable alteration of titanium fouling, which depends on polarization and fluid nature.

For model solutions (calcium and phosphate ions, synthetic ultrafiltrate) under cathodic polarization, an important calcium phosphate deposit is observed (above 25 $\left.\mu \mathrm{g} \cdot \mathrm{cm}^{-2} \cdot \mathrm{min}^{-1}\right)$, which decreases in free fouling conditions. Anodic polarization is characterized by a very small deposit $\left(0,5-1 \mu \mathrm{g} \cdot \mathrm{cm}^{-2} \cdot \mathrm{min}^{-1}\right)$.

Pure soluble protein solutions ( $\alpha$-lactalbumin, $\beta$-lactoglobulin) in the absence of phosphate ions give rise to a very small deposit $\left(1.2-2.2 \mu \mathrm{g} \cdot \mathrm{cm}^{-2} \cdot \mathrm{min}^{-1}\right)$ slightly greater in anodic conditions. Its increase is however considerable in the presence of phosphates and in the cathodic range $\left(17-27 \mu \mathrm{g} \cdot \mathrm{cm}^{-2} \cdot \mathrm{min}^{-1}\right)$.

More complex solutions (milk, whey, sodium caseinate) are characterized by an important increase of deposit, both in cathodic (up to about $50 \mu \mathrm{g} \cdot \mathrm{cm}^{-2} \cdot \mathrm{min}^{-1}$ ) and anodic $\left(8-100 \mu \mathrm{g} \cdot \mathrm{cm}^{-2} \cdot \mathrm{min}^{-1}\right)$ conditions, as compared to free fouling $(2,0-5,0$ $\left.\mu \mathrm{g} \cdot \mathrm{cm}^{-2} \cdot \min ^{-1}\right)$.

Applying an electric field means modifications for electrode phenomena : negative charge of the surface and $\mathrm{pH}$ rise in the vicinity of the cathode, positive surface charge and $\mathrm{pH}$ decrease for the anode. These alterations bring about two types of basic consequences : acceleration of calcium phosphate precipitation (this substance showing a great tendency to get amorphous in such conditions) with a cathodic polarization, and protein precipitation for an anodic polarization.

In the cathodic range, an analysis of transient curves gives informations matching up with qualitative and quantitative aspects of deposit analysis, such as surface covering up, adhesion or isolating capacity. A relationship seems to exist between overpotentials (energy required to carry out oxygen or water reduction), nature, and perhaps mass of deposit. Therefore, overpotential measurements could be a basic principle for a fouling sensor, provided that a few complementary research be made about optimization of electrochemical conditions (current, impulse duration...) and calibration referred to a non fouling fluid.

Lastly, as for the fouling mechanism as a whole, the results evidence an interaction between proteins, phosphates and calcium ions. The poor solubility of complexes built up in this way confirms the most important part played by phosphate ions on the fouling intensity itself.

Key words : Fouling - Heat transfer surface - Electric field - Whey - Milk - Infrared spectrometry - Electron spectrometry. 


\section{Introduction}

Le mécanisme de l'encrassement de surfaces d'échange de chaleur par du lactosérum et du lait, récemment proposé par DAUFIN et al. (1987) peut être résumé de la manière suivante : dès les premiers instants de l'encrassement, des lipides, en faible quantité, ainsi que des protéines, des phosphates et du calcium sont présents sur la surface de l'acier inoxydable. Il semble difficile d'attribuer aux lipides un rôle moteur, tant dans l'élaboration des premières couches que dans la croissance des dépôts. Par contre, les ions calcium constituent un élément-clé. Ils sont en effet susceptibles d'interactions avec les protéines, notamment au niveau des groupements carboxylates des protéines solubles et de la caséine, mais ils donnent également des composés peu solubles avec la base phosphate $\mathrm{PO}_{4}{ }^{3-}$ susceptible, elle, de fournir, en présence d'acides carboxyliques, l'acide conjugué $\mathrm{HPO}_{4}{ }^{2-}$ dont le taux règle la cristallisation en apatite du phosphate de calcium amorphe initial. Il y a donc a priori une interaction triple et la croissance même du dépôt est fondamentalement liée à la présence de calcium et de phosphate qui assurent un lien entre les protéines et stabilisent ce dépôt. En conséquence, l'inhibition de la formation de phosphate de calcium sur une surface d'échange constituera un moyen de lutte contre l'encrassement.

On sait que l'adsorption de certains constituants du lait sur des matériaux métalliques dépend des caractéristiques électrochimiques de l'interface (BeLJELTI et al., 1980 ; IVARSSON et al., 1981 ; FORT, 1983) et que l'application d'un champ électrique modifie les phénomènes à l'électrode (Daufin et al., 1985). Nous nous sommes donc proposés d'appliquer un champ électrique à une surface d'échange de chaleur pour modifier localement les phénomènes dans le but de tenter d'inhiber la formation de phosphate de calcium (donc de réduire l'encrassement) d'une part, et d'apporter des éléments nouveaux pour confirmer ou infirmer le mécanisme précédent d'autre part. Enfin, nous avons tenté d'analyser les phénomènes électrochimiques à l'interface à l'aide d'une méthodologie utilisant des impulsions de courant.

\section{Matériels et méthodes}

\section{A. Plate-forme et conditions d'encrassement}

L'encrassement est réalisé à l'aide d'une installation de laboratoire décrite en détail par Roignant et al. (1983). Elle est constituée d'un préchauffeur tubulaire et d'une maquette d'échange de chaleur dans laquelle le fluide encrassant circule à une vitesse moyenne de $0,09 \mathrm{~m} \cdot \mathrm{s}^{-1}$ (débit volumique = $\left.10 \mathrm{l} \cdot \mathrm{h}^{-1}\right)$. Dans ces conditions, à la température de nos essais $\left(72^{\circ} \mathrm{C}\right)$, le nombre de Reynolds est 540 pour le lactosérum et 380 pour le lait; la contrainte de cisaillement à la paroi est alors respectivement de 0,12 et $0,17 \mathrm{~Pa}$. Le long de l'autre face de l'électrode de travail l'eau distillée circule à contre-courant à $0,35 \mathrm{~m} \cdot \mathrm{s}^{-1}\left(250 \mathrm{l} \cdot \mathrm{h}^{-1}\right)$ à $77^{\circ} \mathrm{C}$, créant ainsi une différence de température de $5^{\circ} \mathrm{C}$ entre les deux fluides (DAufin et al., 1985). 


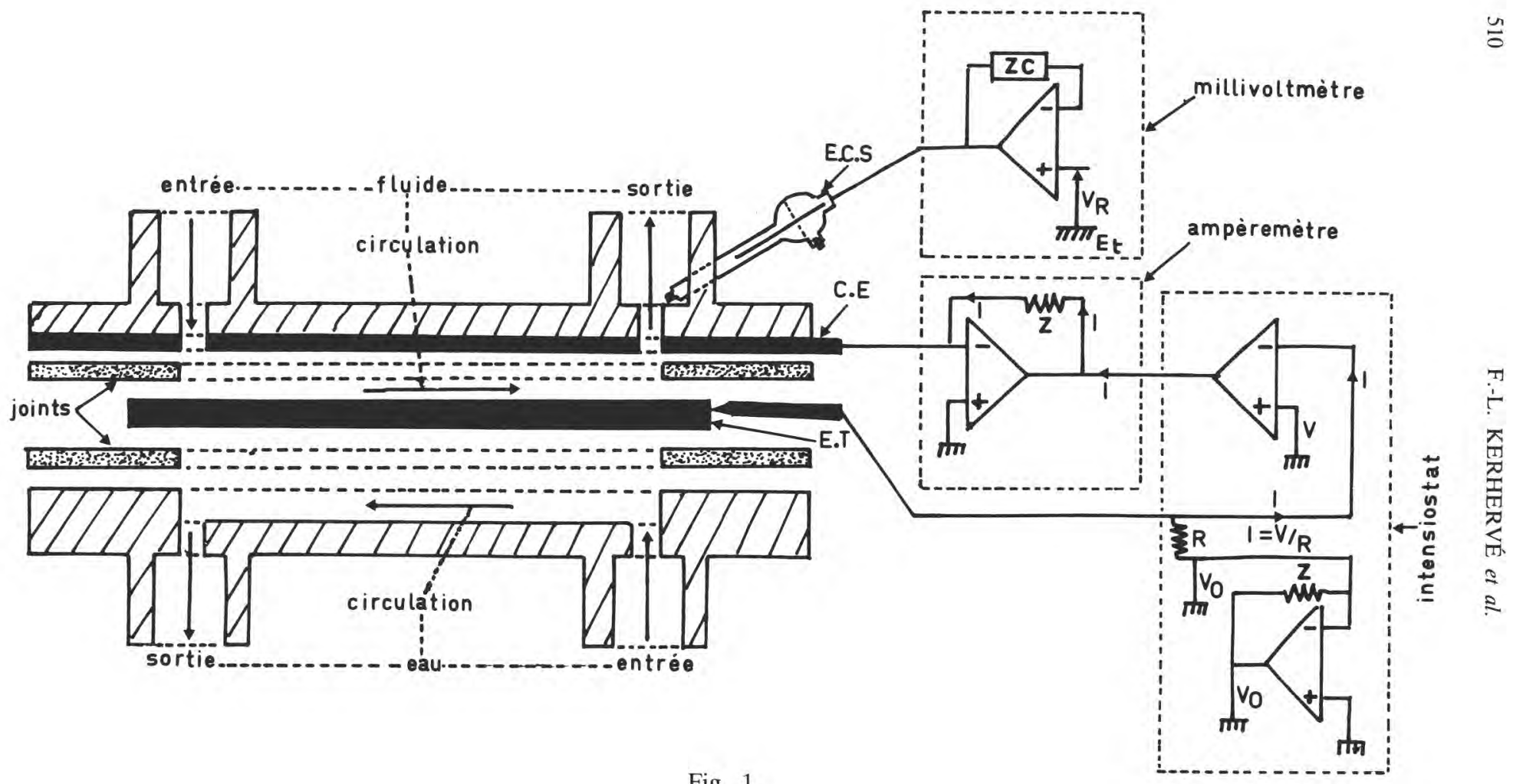

Coupe longitudinale de la maquette d'échange de chaleur: électrodes et montage intensiostatique. (Z: impédance finie; ZC: impédance infinie; $V_{R}:$ tension de référence; $V_{O}:$ potentiel virtuel nul par rapport à $E_{t} ; V:$ tension de consigne; $E_{t}:$ potentiel de la terre; E.C.S. : électrode au calomel saturé; C.E. : contre-électrode; E.T. : électrode de travail ; I : intensité).

Longitudinal section of the heat exchange model : electrodes and intensiostatic assembly diagram. ( $Z$ : finite impedance; ZC : infinite impedance; $V_{R}$ : reference tension; $V_{O}:$ Zero virtual potential versus $E_{t} ; V:$ applied tension ; $E_{t}:$ ground potential ; E.C.S. : saturated calomel electrode ; C.E. : auxiliary electrode; E.T. : working electrode ; $I$ : current intensity). 


\section{B. Montage électrochimique et électrodes}

Pour effectuer les différentes mesures électrochimiques, avec ou sans courant électrique imposé, nous utilisons le montage décrit dans la figure 1. Il est essentiellement constitué d'un intensiostat (Tacussel PRT 10-05), d'un millivoltmètre, d'un ampèremètre et de trois électrodes implantées dans la maquette.

L'électrode de travail est isolée galvaniquement du reste de l'installation par un joint de polytétrafluoréthylène. Elle consiste en une plaque de titane UT40 $(50 \times 30 \times 0,8 \mathrm{~mm})$ laminée à froid, ce matériau étant préféré à l'acier inoxydable en raison de son excellente résistance à la corrosion. L'aire de travail $\left(8 \mathrm{~cm}^{2}\right)$ est polie mécaniquement, sous eau courante (papier de granulométrie $12 \mu \mathrm{m}, \mathrm{n}^{\circ} 800$ ), rincée à l'eau distillée puis séchée à l'air pendant 24 h; pour certains essais, afin de s'affranchir de l'oxydation du titane, les échantillons sont platinés (étalement de couches minces de $\mathrm{H}_{2} \mathrm{PtCl}_{6}, 6 \mathrm{H}_{2} \mathrm{O}$, en présence d'huiles essentielles puis cuisson au four à $450-500{ }^{\circ} \mathrm{C}$ pendant $15-20$ $\mathrm{mn}$, l'opération étant renouvelée 5 fois). Une contre électrode, également en titane, est fixée parallèlement à l'électrode de travail, ce qui permet une distribution homogène des lignes de courant perpendiculairement au matériau étudié. Enfin, une électrode de référence au calomel saturé (E.C.S.) est située à la sortie du canal d'écoulement, ce qui peut entraîner une légère chute ohmique entre l'entrée et la sortie du canal d'écoulement.

\section{Procédure expérimentale}

Avant chaque essai, l'installation est nettoyée selon la séquence suivante : $\mathrm{NaOH} 2 \%, 65^{\circ} \mathrm{C}, 10 \mathrm{mn} ; \mathrm{HNO}_{3} 1,3 \%, 70^{\circ} \mathrm{C}, 5 \mathrm{mn}$, un rinçage à l'eau distillée étant effectué entre les deux produits et à la fin de la séquence. La durée de l'essai d'encrassement est fixée à 5 ou 20 minutes. Trois répétitions au moins sont effectuées sauf dans le cas des solutions d' $\alpha$-lactalbumine et de $\beta$-lactoglobuline pour lesquelles un seul essai est réalisé.

\section{Essais sous courant continu}

Les essais d'encrassement en conditions intensiostatiques sont réalisés sous des densités de courant continu constantes allant jusqu'à $1250 \mu \mathrm{A} \cdot \mathrm{cm}^{-2}$. En anodique, l'électrode de travail est le pôle positif où se produisent les réactions d'oxydation ; en cathodique, cette électrode, négative, est le siège de réactions de réduction. La polarisation de l'électrode à la fin de l'essai est la différence entre le potentiel sous courant imposé et le potentiel en encrassement naturel. Le courant électrique est appliqué dès le début de la phase d'équilibrage hydrodynamique et thermique sur eau distillée puis est maintenu durant l'encrassement par les différents fluides et enfin pendant le rinçage final à l'eau distillée.

\section{Essais sous courant pulsé}

Nous étudions la réponse de l'interface titane-fluide encrassant à l'aide d'une technique intensiostatique du type impulsionnel. Nous enregistrons l'évolution du potentiel électrochimique du titane au cours de l'application d'une 
impulsion anodique ou cathodique de courant valant $125 \mu \mathrm{A} \cdot \mathrm{cm}^{-2}$. Cette mesure de courte durée ( $30 \mathrm{~s})$ qui perturbe donc peu le système, est effectuée au début $(\mathrm{t}=0)$ et à la fin $(\mathrm{t}=20 \mathrm{mn})$ de la séquence d'encrassement afin de pouvoir analyser les différences apparues en raison de la croissance de la couche de dépôt. La surtension est calculée par différence entre les valeurs du potentiel du titane après et avant l'imposition du courant pendant 30 secondes.

Elle est représentative de l'énergie nécessaire pour oxyder ou réduire les substances présentes à l'interface solide-liquide (DIXBY, 1977 ; BARD et FAULKNER, 1983; LANDOLT, 1984 ; TAl et al., 1985). C'est donc une caractéristique globale des phénomènes se produisant à l'interface. Les variations de surtension au cours du temps permettent d'appréhender la chute ohmique due à la résistance de la solution, la charge de la double couche métal-solution, la présence d'un palier faradique correspondant à une réaction électrochimique, l'apparition de phénomènes de diffusion inhérents au transport des espèces en solution et, enfin, l'existence éventuelle d'une seconde réaction électrochimique.

Par comparaison avec un système de référence dont on connaît le comportement, il est donc possible de détecter, en analysant la réponse transitoire : surtension en fonction du temps, les modifications apportées à l'état de surface de l'électrode et, en particulier, son encrassement.

Le montage utilisé est de type intensiostatique classique, le millivoltmètre étant remplacé par un oscilloscope à mémoire numérique (Schlumberger, type 5027) qui stocke la courbe en temps réel et permet de la restituer à vitesse plus lente sur table traçante $\mathrm{X}-\mathrm{Y}$.

\section{Fluides utilisés}

Au cours de cette étude nous avons utilisé des fluides industriels (lait et lactosérum) et des solutions modèles (solution de "phosphate et calcium ", ultrafiltrat synthétique) qui sont présentés dans un travail récent (DAUFIN et $a l$, 1987). Nous avons complété cette gamme de fluides par des solutions de protéines : caséinate en présence de 0 à $800 \mathrm{mg} \cdot \mathrm{l}^{-1}$ d'ions $\mathrm{Ca}^{2+} ; \alpha$-lactalbumine $(\alpha \mathrm{lb})$ solubilisée dans l'ultrafiltrat synthétique contenant ou non calcium et phosphates; $\beta$-lactoglobuline ( $\beta \mathrm{lg}$ ) en présence de 0 ou $400 \mathrm{mg} \cdot \mathrm{l}^{-1}$ de calcium, sans ou avec phosphates. Les compositions chimiques, évaluées à l'aide des méthodes d'analyse décrites précédemment (DAufin et al., 1987), sont indiquées dans le tableau 1.

\section{E. Mesure de l'encrassement}

La masse de dépôt est mesurée par sa pesée, le dépôt lui-même étant analysé par spectrophométrie de photo-électrons X (XPS) et par spectrophotométrie infrarouge (IR) (DAUFIN et al., 1987).

Des hétérogénéités de composition du dépôt, dans le sens d'avancée du fluide sur l'échantillon, ont été constatées expérimentalement ( $\beta I g$ en présence de calcium et de phosphates ; caséinate). Elles imposent, pour la comparaison, d'effectuer les prélèvements de manière identique lorsque le dépôt est suffi- 
TABLEAU 1

Principaux éléments de composition chimique des différents fluides utilisés à pH 6,6

Main chemical components of the fluids used at $\mathrm{pH} 6.6$

\begin{tabular}{|c|c|c|c|c|c|c|c|c|}
\hline \multirow{3}{*}{ Fluide } & \multicolumn{8}{|c|}{ Composition } \\
\hline & \multicolumn{6}{|c|}{$\left({\mathrm{g} . \mathrm{kg}^{-1}}^{-1}\right)$} & \multicolumn{2}{|c|}{$\left(\mathrm{mg} \cdot \mathrm{kg}^{-1}\right)$} \\
\hline & $\begin{array}{l}\text { Matières } \\
\text { sèches }\end{array}$ & Protéines & Caséine & $\begin{array}{c}\alpha \text {-lact- } \\
\text { albumine }\end{array}$ & $\begin{array}{l}\beta \text {-lacto- } \\
\text { globuline }\end{array}$ & Lactose & $\begin{array}{c}\text { Calcium } \\
\text { total }\end{array}$ & $\begin{array}{c}\text { Phosphore } \\
\text { total }\end{array}$ \\
\hline «Phosphate + calcium » & 3,6 & 0 & 0 & 0 & 0 & 0 & 400 & 400 \\
\hline Ultrafiltrat "synthétique » & 58,9 & 0 & 0 & 0 & 0 & 49,5 & 400 & 400 \\
\hline Ultrafiltrat modifié & 3,2 & 0 & 0 & 0 & 0 & 0 & $0 *$ & $0 *$ \\
\hline Solutions d' $\alpha$-lactalbumine $* *$ & $3,4-4,1$ & $0,24-0,70$ & 0 & $0,24-0,70$ & 0 & $0-49,5$ & $0-800$ & $0-400$ \\
\hline Solutions de $\beta$-lactoglobuline $* *$ & $4,2-4,8$ & 1,0 & 0 & 0 & 1,0 & 0 & $0-400$ & $0-400$ \\
\hline Solutions de caséinate & 25,0 & 22,7 & 22,7 & 0 & 0 & 0 & $0-800$ & 220 \\
\hline Lactosérum & 63,2 & 13,2 & 0 & 0,9 & 4,0 & 49 & 300 & 370 \\
\hline Lait & 90,8 & 34,7 & 25,6 & 1,1 & 3,6 & 49,8 & 1255 & 930 \\
\hline
\end{tabular}

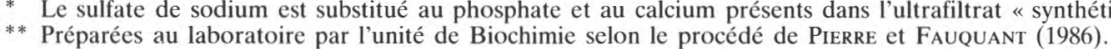


sant, de ne tenir compte que des tendances générales dans le cas contraire. L'analyse locale, inversement, fournit un paramètre supplémentaire d'appréciation.

\section{Résultats}

Les masses de dépôt sont exprimées en $\mu \mathrm{g} \cdot \mathrm{cm}^{-2} \cdot \mathrm{mn}^{-1}$, car l'encrassement est un phénomène qui évolue dans le temps. Toutefois, l'extrapolation des vitesses de dépôts à des durées supérieures à celles de nos essais (5 à 20 minutes) doit être faite avec prudence car la cinétique de formation du dépôt n'est pas linéaire.

Les variations de masses de dépôts en fonction du courant imposé sont représentées sur la figure 2 pour le lait, le lactosérum et les solutions de caséinate et sur la figure 3 pour les autres fluides : « $\mathrm{PO}_{4}+\mathrm{Ca} »$, ultrafiltrat, solutions d' $\alpha$-lactalbumine et de $\beta$-lactoglobuline.

Pour le lait et le lactosérum, les masses obtenues en anodique et en cathodique sont plus fortes qu'en encrassement libre (fig. 2a).

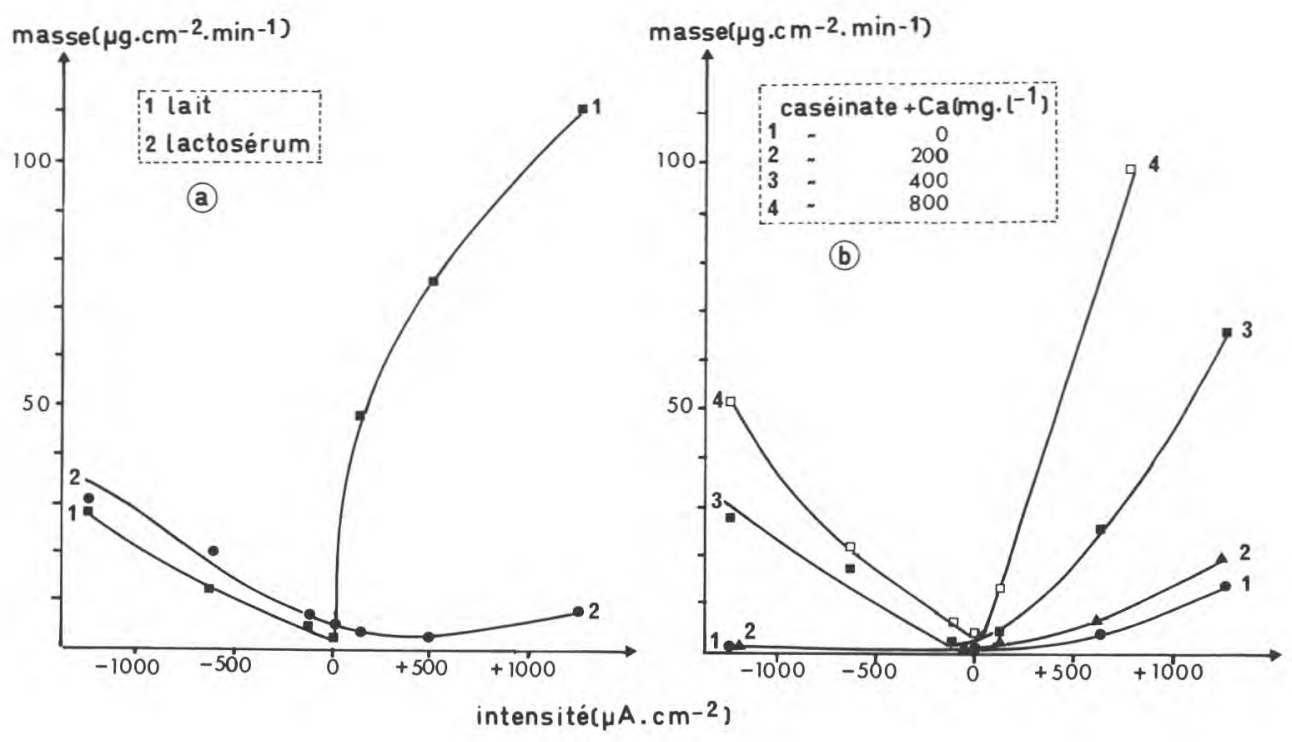

Fig. 2

Influence de l'intensité imposée sur la masse de dépôt sur le titane après contact avec du lactosérum, du lait ou des solutions de caséinate $\left(72^{\circ} \mathrm{C}, \Delta T=5{ }^{\circ} \mathrm{C}, 20 \mathrm{mn}\right)$.

Effect of controlled intensity on deposit mass : titanium plate in contact with whey, milk or caseinate solutions $\left(72^{\circ} \mathrm{C}, \Delta T=5^{\circ} \mathrm{C}, 20 \mathrm{~min}\right)$. 


\section{A. Lactosérum}

L'analyse du dépôt par XPS montre que la proportion de calcium et de phosphore est plus faible en encrassement libre que sous polarisation cathodique (tabl. 2). Par contre, sous polarisation anodique, $\mathrm{Ca}$ et $\mathrm{P}$ disparaissent. L'analyse IR confirme ces résultats : en cathodique la proportion de protéines diminue tandis que celle des. phosphates augmente; sous polarisation anodique les phosphates disparaissent à peu près totalement (tabl. 2). Lorsque la durée augmente de 5 à $20 \mathrm{mn}$ on observe une augmentation de la proportion des phosphates (phase toujours amorphe vérifiée aux rayons X). Il faut aussi noter qu'en l'absence de courant électrique imposé, l'abaissement du $\mathrm{pH}$ par ajout d' $\mathrm{HCl}$ au sein du fluide conduit à des résultats comparables à ceux obtenus dans le domaine anodique. En effet, lorsque le $\mathrm{pH}$ passe de 6,6 à 5,8, la proportion des phosphates diminue très fortement (de $20 \%$ à $3 \%$ ) par rapport aux protéines.

TABLEAU 2

Analyse des dépôts par le lactosérum et le lait $\left(72{ }^{\circ} \mathrm{C}, \Delta T=5^{\circ} \mathrm{C}\right)$ en encrassement naturel $(O)$ ou sous courant imposé $\left(625 \mu \mathrm{A} \cdot \mathrm{cm}^{-2}\right)$ cathodique $(C)$ et anodique $(A)$

Analysis of deposits by whey and milk $\left(72{ }^{\circ} \mathrm{C}, \Delta T=5{ }^{\circ} \mathrm{C}\right)$ in free fouling conditions $(O)$ and under cathodic $(C)$ or anodic $(A)$ current $\left(625 \mu \mathrm{A.cm}{ }^{-2}\right)$

\begin{tabular}{|c|c|c|c|c|c|c|c|c|}
\hline \multirow{2}{*}{$\begin{array}{c}\text { Nature } \\
\text { du fluide }\end{array}$} & \multicolumn{4}{|c|}{ XPS (\% en nombre d'atomes) ${ }^{\mathrm{a}}$} & \multicolumn{4}{|c|}{ IR ( $\%$ en nombre de liaisons) $)^{b}$} \\
\hline & $\begin{array}{c}\text { Elément } \\
\text { dosé }\end{array}$ & C & $\mathrm{O}$ & A & $\begin{array}{c}\text { Elément } \\
\text { dosé }\end{array}$ & $\mathrm{C}$ & $\mathrm{O}$ & A \\
\hline $\begin{array}{c}\text { Lactosérum } \\
5 \mathrm{mn}\end{array}$ & $\begin{array}{c}\mathrm{C} \\
\mathrm{O} \\
\mathrm{N} \\
\mathrm{Ca} \\
\mathrm{P}\end{array}$ & & $\begin{array}{c}(0,7) \\
80 \\
13 \\
6 \\
- \\
-\end{array}$ & & $\begin{array}{l}\text { Protéines } \\
\mathrm{PO}_{4}^{3-}\end{array}$ & $\begin{array}{c}(5,1) \\
69 \\
31\end{array}$ & $\begin{array}{c}(0,7) \\
88 \\
12\end{array}$ & $\begin{array}{r}(0,9) \\
100 \\
0\end{array}$ \\
\hline $\begin{array}{l}\text { Lactosérum } \\
20 \mathrm{mn}\end{array}$ & $\begin{array}{c}\mathrm{C} \\
\mathrm{O} \\
\mathrm{N} \\
\mathrm{Ca} \\
\mathrm{P}\end{array}$ & $\begin{array}{c}(17,0) \\
51 \\
30 \\
11 \\
6 \\
2\end{array}$ & $\begin{array}{c}(4,8) \\
64 \\
20 \\
11 \\
4 \\
1\end{array}$ & $\begin{array}{c}(3,2) \\
85 \\
7 \\
8 \\
0 \\
0\end{array}$ & $\begin{array}{l}\text { Protéines } \\
\mathrm{PO}_{4}{ }^{3-}\end{array}$ & $\begin{array}{c}(22,5) \\
57 \\
43\end{array}$ & $\begin{array}{c}(4,0) \\
82 \\
18\end{array}$ & $\begin{array}{c}(3,2) \\
99 \\
1\end{array}$ \\
\hline $\begin{array}{l}\text { Lait } \\
20 \mathrm{mn}\end{array}$ & $\begin{array}{c}\mathrm{C} \\
\mathrm{O} \\
\mathrm{N} \\
\mathrm{Ca} \\
\mathrm{P}\end{array}$ & $\begin{array}{c}(13,2) \\
52 \\
29 \\
8 \\
8 \\
3\end{array}$ & $\begin{array}{c}(6,0) \\
43 \\
48 \\
7 \\
2 \\
\text { traces }\end{array}$ & $\begin{array}{c}(81,0) \\
83 \\
5 \\
11 \\
0 \\
1\end{array}$ & $\begin{array}{l}\text { Protéines } \\
\mathrm{PO}_{4}{ }^{3-}\end{array}$ & $\begin{array}{c}(17,1) \\
92 \\
8\end{array}$ & $\begin{array}{c}(5,6) \\
95 \\
5\end{array}$ & $\begin{array}{c}(70,0) \\
99 \\
1\end{array}$ \\
\hline
\end{tabular}

a :\% rapporté à la somme des nombres d’atomes : Carbone $(\mathrm{C})+$ Oxygène $(\mathrm{O})+$ Azote $(\mathrm{N})+\mathrm{Calcium} .(\mathrm{Ca})+$ Phosphore (P).

$\mathrm{b}: \%$ rapporté à la somme des nombres de liaisons : Protéines + Phosphates $\left(\mathrm{PO}_{4}{ }^{3-}\right)$ les lipides, bien que présents, ne modifient pas les variations relatives des protéines et des phosphates. Ils ne sont donc pas pris en compte.

(...) masse de dépôt en $\mu \mathrm{g} \cdot \mathrm{cm}^{-2} \cdot \mathrm{mn}^{-1}$. 


\section{B. Lait}

Comparées aux masses obtenues avec le lactosérum, celles mesurées avec le lait sont un peu plus faibles en cathodique mais considérablement plus élevées en anodique (fig. 2a).

L'analyse XPS du dépôt révèle les mêmes tendances que pour le lactosérum (tabl. 2) : en cathodique les pourcentages de $\mathrm{Ca}$ et de $\mathrm{P}$ augmentent ; en anodique, Ca disparaît alors que l'on retrouve toujours un peu de P. L'analyse IR confirme également ces résultats : sous polarisation cathodique, le pourcentage de protéines diminue alors que celui des phosphates augmente; sous contrôle anodique, le dépôt est presque exclusivement formé de protéines (tabl. 2).

\section{Solution de phosphate et de calcium}

Le dépôt formé [hydrogénophosphate $\mathrm{Ca}_{4} \mathrm{H}\left(\mathrm{PO}_{4}\right)_{3}, 2-3 \mathrm{H}_{2} \mathrm{O}$ ] a la même structure (vue en IR) en cathodique et en encrassement libre. Son adhérence au support est très faible: il se détache très facilement; par contre, en anodique, le profil du spectre observé est original et la présence de phosphate de titane n'y est pas exclue.

\section{Ultrafiltrat synthétique}

Les masses sont du même ordre de grandeur que celles obtenues avec le lactosérum (fig. 3a).

L'analyse du dépôt par IR montre, outre les absorptions caractéristiques des phosphates majoritaires, les deux bandes des carboxylates vers 1600 et $1400 \mathrm{~cm}^{-1}$. On a donc vraisemblablement des citrates dont la proportion, par rapport aux phosphates, est plus importante pour l'essai en cathodique que pour le témoin, le rapport des bandes à 1400 et $1050 \mathrm{~cm}^{-1}$ étant deux fois plus grand. Au niveau structural, le phosphate de calcium, de type apatite cryptocristalline pour le témoin, devient amorphe, très hydraté, en cathodique. Enfin, les milieux anodiques posent le même problème que pour la solution de phosphate et de calcium : c'est toujours le même profil particuiier pour la bande de phosphates que l'on observe vers $1100 \mathrm{~cm}^{-1}$ avec la même possibilité d'intervention de phosphate de titane.

\section{E. Solutions d' $\alpha$-lactalbumine}

Avec les solutions d' $\alpha$-lactalbumine, l'analyse IR n'est pas interprétable pour des dépôts aussi faibles (quelques $\mu \mathrm{g} \cdot \mathrm{cm}^{-2} \cdot \mathrm{mn}^{-1}$; fig. 3a) : l'influence de la pollution n'est pas contrôlable.

En présence de phosphate et de calcium dans la solution d' $\alpha$-lactalbumine, les masses deviennent très élevées en cathodique $\left(17 \mu \mathrm{g} \cdot \mathrm{cm}^{-2} \cdot \mathrm{mn}^{-1}\right.$; fig. $\left.3 \mathrm{a}\right)$.

\section{F. Solutions de $\beta$-lactoglobuline}

Avec les solutions de $\beta$-lactoglobuline sans calcium ni phosphate, l'analyse XPS révèle qu'en cathodique le dépôt, faible, est exclusivement organique 


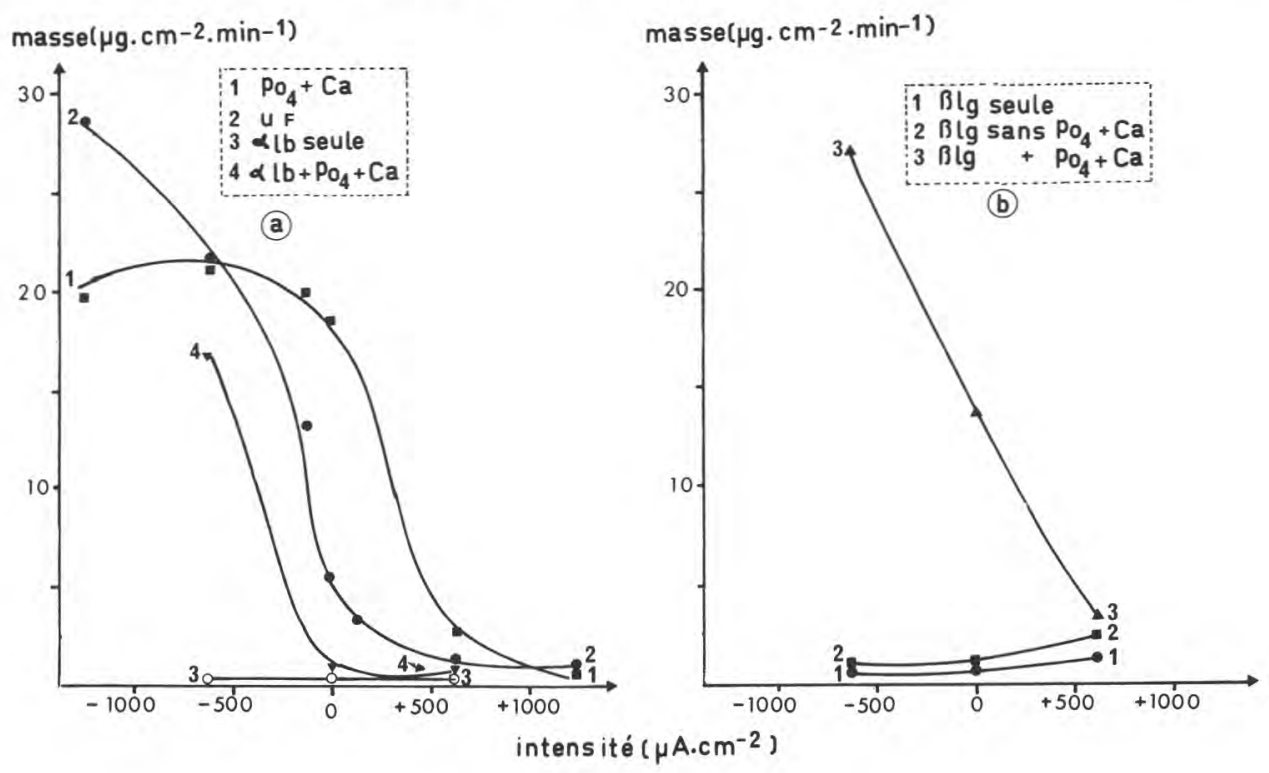

Fig. 3

Influence de l'intensité imposée sur la masse de dépôt sur le titane après contact avec l'ultrafiltrat $(U F)$, une solution de " $\mathrm{PO}_{4}+\mathrm{Ca}$, des solutions d' $\alpha$-lactalbumine $(\alpha l b)$ ou de $\beta$-lactoglobuline $(\beta \mathrm{lg})\left(72{ }^{\circ} \mathrm{C}, \Delta T=5{ }^{\circ} \mathrm{C}, 20 \mathrm{mn}\right)$.

Effect of controlled intensity on deposit mass : titanium plate in contact with ultrafiltrate (UF), « $\mathrm{PO}_{4}+\mathrm{Ca} » \alpha$-lactalbumin $(\alpha \mathrm{lb})$ or $\beta$-lactoglobulin ( $\beta$ lg) solutions $\left(72{ }^{\circ} \mathrm{C}\right.$, $\Delta T=5^{\circ} \mathrm{C}, 20 \mathrm{~min}$ ).

tandis que l'IR ne peut fournir aucun renseignement fiable, pour les mêmes raisons que précédemment.

L'addition de calcium accroît les masses, surtout en anodique (fig. 3b) et les dépôts paraissent contenir exclusivement des protéines. Il est difficile de conclure quant à leur structure en raison, notamment, d'une mauvaise répétabilité des expériences.

Enfin, l'addition de phosphates à la solution précédente augmente très fortement les masses en encrassement libre et en cathodique (fig. 3b). De plus, toutes les masses sont supérieures à celles obtenues avec l'UF (fig. 3a).

L'analyse IR montre que la proportion de phosphates par rapport aux protéines est de $65 \%$ minimum (phase apatite) pour le témoin. Elle augmente normalement en conditions cathodiques ( $90 \%$ au moins, phase amorphe) pour devenir très faible en conditions anodiques (2\%) (tabl. 3). La présence de citrates est tout aussi vraisemblable que pour l'UF lui-même dans les deux 
TABLEAU 3

Analyse des dépôts par les solutions de caséinate de sodium et de $\beta$-lactoglobuline $\left(72{ }^{\circ} \mathrm{C}\right.$, $\left.\Delta T=5^{\circ} \mathrm{C}, 20 \mathrm{mn}\right)$ en encrassement naturel $(O)$ ou sous courant imposé $\left(625 \mu \mathrm{A} . \mathrm{cm}^{-2}\right)$ cathodique $(C)$ et anodique $(A)$

Analysis of deposits by sodium caseinate and $\beta$-lactoglobulin solutions ( 72 " $C$, $\left.\Delta T=5{ }^{\circ} \mathrm{C}, 20 \mathrm{~min}\right)$ in free fouling solutions $(O)$ and under cathodic $(C)$ or anodic $(A)$ current $\left(625 \mu \mathrm{A} \cdot \mathrm{cm}^{-2}\right)$

\begin{tabular}{|c|c|c|c|c|c|c|c|c|}
\hline \multirow{2}{*}{$\begin{array}{l}\text { Nature } \\
\text { du fluide }\end{array}$} & \multicolumn{4}{|c|}{ XPS (\% en nombre d'atomes) ${ }^{a}$} & \multicolumn{4}{|c|}{ IR ( $\%$ en nombre de liaisons $)^{\mathrm{b}}$} \\
\hline & $\begin{array}{l}\text { Elément } \\
\text { dosé }\end{array}$ & $\mathrm{C}$ & $\mathrm{O}$ & A & $\begin{array}{l}\text { Elément } \\
\text { dosé }\end{array}$ & $\mathrm{C}$ & $\mathrm{O}$ & A \\
\hline Caséinate & & & & & & & & \\
\hline & $\mathrm{C}$ & $\begin{array}{c}(0,5) \\
60\end{array}$ & $\begin{array}{l}(0,4) \\
65\end{array}$ & $\begin{array}{c}(5,5) \\
74\end{array}$ & & & & \\
\hline $\mathrm{PO}_{4}: 0$ & $\mathrm{O}$ & 28 & 20,5 & 11 & & & & \\
\hline & $\mathrm{N}$ & 12 & 14,5 & 15 & & & & \\
\hline $\mathrm{Ca}: 0$ & $\begin{array}{l}\mathrm{Ca} \\
\mathrm{P}\end{array}$ & 0 & 0 & 0 & & & & \\
\hline & 1 & $(16,5)$ & $(2,1)$ & $(17,8)$ & & $(18,6)$ & $(1,6)$ & $(26,8)$ \\
\hline $\mathrm{PO}_{4}: 0$ & $\begin{array}{l}\mathrm{C} \\
\mathrm{O}\end{array}$ & $\begin{array}{l}68 \\
15\end{array}$ & 19 & 12 & Protéines & 99 & 100 & 100 \\
\hline $\mathrm{Ca} \cdot 400 \mathrm{mg} \mathrm{l}^{-1}$ & $\mathrm{~N}$ & $\begin{array}{r}15 \\
2\end{array}$ & $\begin{array}{c}14 \\
\text { traces }\end{array}$ & 10 & $\mathrm{PO}_{4}$ & 1 & 0 & 0 \\
\hline Ca : $400 \mathrm{mg} .1$ & P & $\begin{array}{l}2 \\
0\end{array}$ & $\begin{array}{c}\text { traces } \\
0\end{array}$ & & & & & \\
\hline $\mathrm{PO}_{4}: 0$ & & & & & Protéines & $\begin{array}{c}(21,3) \\
98\end{array}$ & $\begin{array}{c}(3,2) \\
100\end{array}$ & $\begin{array}{l}(49) \\
100\end{array}$ \\
\hline $\mathrm{Ca}: 800 \mathrm{mg} \cdot \mathrm{l}^{-1}$ & & & & & $\mathrm{PO}_{4}$ & 2 & 0 & 0 \\
\hline$\beta$-lactoglobuline & & & & & & & & \\
\hline $\begin{array}{l}\mathrm{PO}_{4}: 0 \\
\mathrm{Ca}: 0\end{array}$ & $\begin{array}{l}\mathrm{C} \\
\mathrm{O} \\
\mathrm{N}\end{array}$ & $\begin{array}{c}(0,1) \\
52 \\
25 \\
14\end{array}$ & & & & & & \\
\hline & & $(0,7)$ & & & & $(2,1)$ & $(2,5)$ & $(31,3)$ \\
\hline $\mathrm{PO}_{4}: 0$ & $\mathrm{C}$ & 58 & & & Protéines & 100 & 100 & 100 \\
\hline $\mathrm{Ca}: 400 \mathrm{mg} . \mathrm{I}^{-1}$ & $\begin{array}{l}\mathrm{O} \\
\mathrm{N}\end{array}$ & $\begin{array}{l}20 \\
22\end{array}$ & & & $\mathrm{PO}_{4}$ & 0 & 0 & 0 \\
\hline & & & & & & $(27)$ & $(13,4)$ & $(3,2)$ \\
\hline $\begin{array}{l}\mathrm{PO}_{4}: 1226 \mathrm{mg} . \mathrm{I}^{-1} \\
\mathrm{Ca}: 400 \mathrm{mg} \cdot \mathrm{I}^{-1}\end{array}$ & & & & & $\begin{array}{l}\text { Protéines } \\
\mathrm{PO}_{4}\end{array}$ & $\begin{array}{l}<10^{* *} \\
>90\end{array}$ & $\begin{array}{l}<35^{* \cdots} \\
>65\end{array}$ & $\begin{array}{r}98 \\
2\end{array}$ \\
\hline
\end{tabular}

(...) masse de dépôt en $\mu \mathrm{g} \cdot \mathrm{cm}^{-2} \cdot \mathrm{mn}^{-1}$ - ** Interférence avec vas $\mathrm{COO}^{-}$des citrates.

a : \% rapporté à la somme des nombres d'atomes : Carbone $(\mathrm{C})+$ Oxygène $(\mathrm{O})+$ Azote $(\mathrm{N})+$ Calcium $(\mathrm{Ca})+$ Phosphore (P).

b:\% rapporté à la somme des nombres de liaisons : protéines + phosphates (PO $\mathrm{P}_{4}$ ). Les lipides bien que présents, ne modifient pas les variations relatives des protéines et des phosphates. Ils ne sont donc pas pris en compte. 
premières conditions, la variation du rapport $\gamma$ d'absorbances à 1400 à 1050 $\mathrm{cm}^{-1}$ étant pratiquement identique $(\gamma=0,05$ pour le témoin, $\gamma=0,1$ en conditions cathodiques). Il faut ainsi noter que le calcul n'a qu'une signification limitée : l'essentiel de l'absorbance à $1650 \mathrm{~cm}^{-1}$ est assurée par la bande de valence antisymétrique $v_{\text {as }} \mathrm{COO}^{-}$des citrates. L'interférence est maximale en cathodique et les vibrations $\mathrm{C}-\mathrm{H}$ de la $\beta \mathrm{lg}$ ne sont alors pas discernables vers $2900-3000 \mathrm{~cm}^{-1}$. La teneur en protéines, ainsi calculée, représente en fait une valeur maximale: les phosphates sont toujours largement majoritaires.

\section{G. Solutions de caséinate de sodium}

Les masses de dépôt, d'autant plus importantes, que la teneur en calcium est élevée (fig. 2b), augmentent à la fois en cathodique et en anodique. Pour obtenir des masses comparables à celles du lait, une teneur supérieure en calcium est nécessaire en anodique : 800 au lieu de $400 \mathrm{mg} \cdot \mathrm{l}^{-1}$ en cathodique.

L'analyse XPS révèle que lorsque la solution de caséinate ne contient ni $\mathrm{PO}_{4}$, ni $\mathrm{Ca}$, le dépôt est uniquement formé de protéines, aussi bien en encrassement libre que sous courant imposé (tabl. 3). Par contre, en présence de calcium, on en retrouve dans le dépôt obtenu en encrassement libre et en cathodique. En outre, de nouvelles analyses XPS effectuées après des érosions successives du dépôt (par bombardement d'ions Argon: Daufin et al., 1987) précisent que la teneur en calcium est plus forte près de la surface métallique ( $2 \%$ en cathodique, $1 \%$ en encrassement libre) qu'à la superficie du dépôt (traces). Ces résultats ne peuvent être confirmés par l'analyse IR qui n'est pas sensible au calcium ; les traces $(2 \%)$ de phosphates mesurés sous polarisation cathodique ne sont pas non plus significatives (tabl. 3).

\section{H. Résultats électrochimiques}

Les polarisations (mesurées lors des essais de $20 \mathrm{mn}$ sous courant imposé) sont indépendantes de l'encrassement. Cependant, en anodique, elles sont beaucoup plus élevées, vraisemblablement en raison de l'oxydation du titane. On a pu s'affranchir de ce phénomène en réalisant quelques essais en anodique $\left(1250 \mu \mathrm{A} . \mathrm{cm}^{-2}\right)$ avec du titane platiné. Les valeurs de polarisation atteintes sont alors du même ordre de grandeur qu'en cathodique mais sont nettement plus faibles que pour le titane. Les masses obtenues sont généralement voisines sur les deux matériaux, mais, dans le cas du lait, beaucoup plus élevées sur titane $\left(110 \mu \mathrm{g} \cdot \mathrm{cm}^{-2} \cdot \mathrm{mn}^{-1}\right)$ que sur titane platiné (32 $\left.\mu \mathrm{g} . \mathrm{cm}^{-2} \cdot \mathrm{mn}^{-1}\right)$. Le $\mathrm{pH}$ atteint sur le premier matériau est vraisemblablement plus faible que sur le second, en raison d'une légère corrosion de $\mathrm{Ti}$, avec apparition locale d'acides de Brönsted tels que $\mathrm{Ti}^{3+}$ et $\mathrm{Ti}^{4+}$, entraînant ainsi une précipitation plus importante des protéines avec formation d'oxydes (ou phosphates) hydratés de titane.

Lors des essais sous courant pulsé anodique, on constate que les surtentions sont plus fortes après $20 \mathrm{mn}$ d'encrassement qu'à l'instant initial mais l'allure des variations des surtensions en fonction du temps et les valeurs mesurées sont indépendantes des fluides. La croissance uniforme d'une couche d'oxyde ou de phosphate de titane explique ces résultats qui ne pourront donc pas être interprétés en relation avec l'encrassement. Dans le domaine cathodi- 
que, les surtensions à l'instant initial sont voisines $(-770 \mathrm{mV})$ pour tous les fluides sauf pour l'UF (fig. 4). Après 20 minutes d'encrassement naturel, la solution de « $\mathrm{PO}_{4}+\mathrm{Ca} »$ conduit à une surtension aussi faible $(-970 \mathrm{mV})$ que celle de l'UF sans $\mathrm{PO}_{4}(-1020 \mathrm{mV})$ tandis que celle obtenue avec le lactosérum $(-1150 \mathrm{mV})$ se rapproche de la valeur mesurée avec l'UF $(-1250 \mathrm{mV})$. L'allure des courbes révèle deux types de comportements: pour l'UF sans phosphate et la solution de « $\mathrm{PO}_{4}+\mathrm{Ca} »$, les courbes réalisées avant encrassement (à l'instant initial) présentent un minimum ; celles réalisées après 20 minutes d'encrassement tendent vers une limite. Pour l'UF et le lactosérum, les courbes avant encrassement, tendent vers une limite ; après 20 minutes d'encrassement, elles présentent un palier.
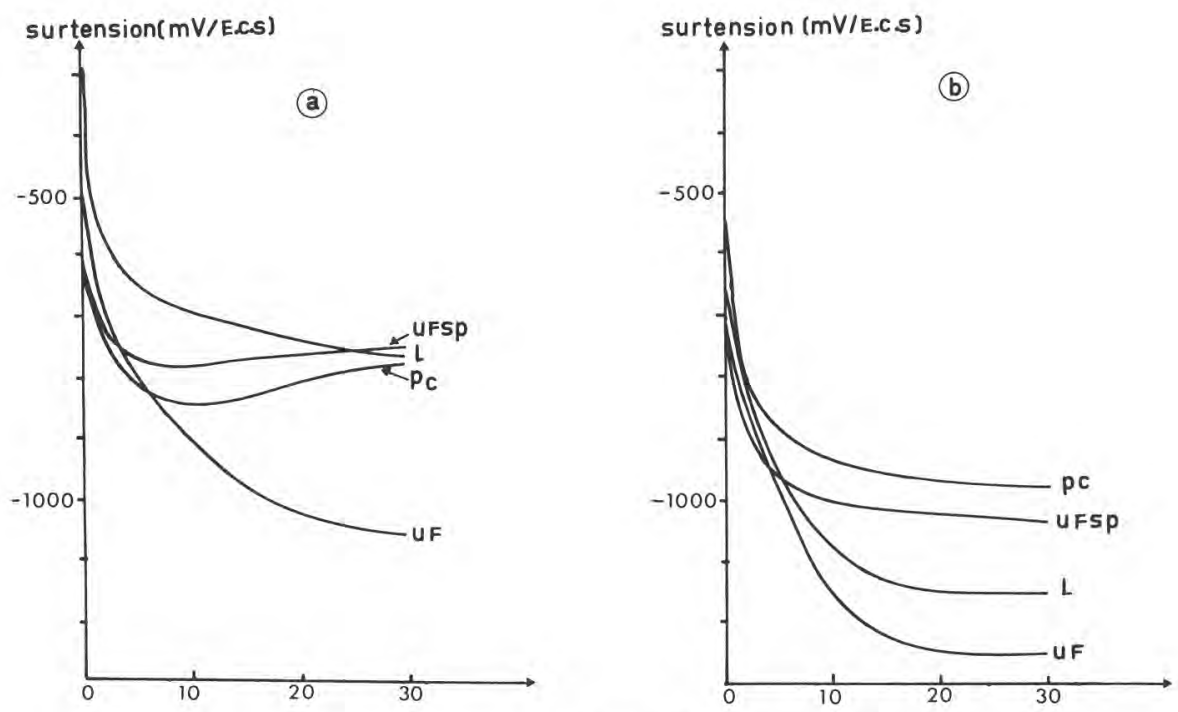

durée(secondes)

Fig. 4

Evolution, en fonction du temps, de la surtension du titane, sous impulsion de courant cathodique (-125 $\left.\mu \mathrm{A} . \mathrm{cm}^{-2}\right)$ pour quelques fluides : ultrafiltrat sans phosphate (UFSP), solution de Phosphate + Calcium $(P C)$, ultrafiltrat $(U F)$, lactosérum $(L)\left(72{ }^{\circ} \mathrm{C}\right.$, $\left.\Delta T=5{ }^{\circ} \mathrm{C}, 20 \mathrm{mn}\right)$.

a. impulsion appliquée à l'instant initial de l'encrassement.

b. impulsion appliquée après $20 \mathrm{mn}$ d'encrassement.

Titanium overpotential vs. time after a cathodic current impulse $\left(-125 \mu \mathrm{A} . \mathrm{cm}^{-2}\right)$ for several fluids : phosphate free ultrafiltrate (UFSP), "Phosphate + Calcium " solution $(P C)$, ultrafiltrate $(U F)$, whey $(L)\left(72{ }^{\circ} \mathrm{C}, \Delta T=5{ }^{\circ} \mathrm{C}, 20 \mathrm{~min}\right)$.

a. impulse applied at initial fouling time.

b. impulse applied after $20 \mathrm{~min}$ fouling. 


\section{Discussion}

L'application d'un champ électrique à un matériau métallique modifie qualitativement et quantitativement le comportement de ce dernier vis-à-vis de l'encrassement. Ces modifications sont différentes suivant le sens de la polarisation et la nature des fluides. En anodique, elles réduisent considérablement l'encrassement par les fluides simples (notamment les solutions de « $\mathrm{PO}_{4}+\mathrm{Ca}$ » et dans une moindre mesure l'UF synthétique) et même par le lactosérum, mais seulement sous certaines intensités anodiques. Mais pour les fluides complexes, tels le lait et le lactosérum, nous obtenons généralement, au contraire, un accroissement du dépôt. Ceci confirme d'ailleurs dans une certaine mesure les résultats de Grasshof (1985) qui n'a pas observé d'effet bénéfique d'un champ électrique (différence de potentiel variant de 250 à 730 $\mathrm{mV}$ ) sur l'encrassement d'un échangeur de chaleur par du lait. Ces variations, peu encourageantes pour réduire l'encrassement, permettent cependant d'expliquer ces résultats et fournissent des éléments nouveaux pour interpréter les mécanismes mis en jeu.

De manière générale en effet, une polarisation cathodique crée, au voisinage immédiat de l'électrode, un gradient de concentration pour les ions; les cations tel que $\mathrm{Ca}^{2+}$ tendent à migrer vers la surface négative où l'élévation du $\mathrm{pH}$ (réduction de l'oxygène dissous ou de l'eau : $2 \mathrm{H}_{2} \mathrm{O}+\mathrm{O}_{2}+4 \mathrm{e}^{-} \rightarrow 4 \mathrm{OH}^{-}$ ou $2 \mathrm{H}_{2} \mathrm{O}+2 \mathrm{e}^{-} \rightarrow \mathrm{H}_{2}+2 \mathrm{OH}^{-}$) déplace les équilibres acido-basiques vers la formation de bases telles que $\mathrm{PO}_{4}{ }^{3-}$ à partir des espèces plus acides telles que $\mathrm{HPO}_{4}{ }^{2-}$ et $\mathrm{H}_{2} \mathrm{PO}_{4}{ }^{-}$. Ces dernières espèces restent d'ailleurs largement majoritaires au sein de la solution. C'est au niveau le plus favorable de ce double gradient que se formera en priorité une espèce phosphate de calcium dont la nature dépend en outre, à température constante, de la composition du fluide. De plus, les différences de mobilité des ions vont entraîner des variations locales de force ionique (par exemple, en présence d'assez fortes quantités d'ions $\left.\mathrm{Ca}^{2+}\right)$. Des effets symétriques s'observent dans le domaine anodique, en particulier acidification locale (oxydation de l'eau : $2 \mathrm{H}_{2} \mathrm{O} \rightarrow \mathrm{O}_{2}+4 \mathrm{H}^{+}+4 \mathrm{e}^{-}$) diminuant la concentration des bases $\left(\mathrm{PO}_{4}{ }^{3-}\right)$. Seuls des phosphates très peu solubles (Ti) peuvent alors se trouver au contact de l'électrode. L'abaissement du $\mathrm{pH}$ limite donc la précipitation des phosphates mais permet d'atteindre le $\mathrm{pH}$ isoélectrique des protéines, favorisant ainsi leur précipitation. L'abaissement volontaire du $\mathrm{pH}$ de 6,6 à 5,8 au sein du fluide (lactosérum) confirme d'ailleurs la diminution importante des phosphates par rapport aux protéines, lesquelles précipitent plus aisément. Une autre conséquence de ces phénomènes est une variation de composition des dépôts dans leur épaisseur du fait que, au cours de la croissance, ils se forment plus loin de l'électrode. La proportion croissante de phosphates par rapport aux protéines, lorsque la durée d'encrassement par le lactosérum passe de 5 à $20 \mathrm{mn}$, en est une illustration marquante. En outre, l'analyse IR effectuée sur un prélèvement en partie supérieure du dépôt obtenu avec le fluide $\beta$-lactoglobuline $+\mathrm{PO}_{4}+\mathrm{Ca}$, en conditions anodiques, montre que la proportion de phosphates $(50,5 \%)$ est alors considérablement supérieure à ce qu'elle est en moyenne dans la totalité de l'épaisseur (tabl. 3). La nucléation hétérogène d'un phosphate de calcium est normalement plus aisée sur une surface comportant déjà ce matériau. 
L'UF montre l'intervention d'espèces carboxylates, normalement plus importantes en conditions cathodiques, et donc une interaction de ces groupements avec les apatites, conduisant à l'analogue des apatites carbonatées obtenues en milieu aqueux (Le Duigou, 1982). Le passage à une phase amorphe en polarisation cathodique (sursaturation locale plus importante) va dans le même sens de moindre cristallinité des apatites carbonatées quand la proportion de carbonate augmente (Legeros et al., 1967).

Pour le lactosérum, qui contient en plus des protéines, l'interaction précédente doit subsister, mais elle intéresse aussi les groupements carboxylates des acides glutamique et aspartique. Le caractère toujours amorphe du phosphate n'est a priori pas étonnant. Quant au profil particulier des variations de masse du dépôt, il est la conséquence de deux effets antagonistes: dịminution de la quantité de phosphates en allant des milieux cathodiques vers les milieux anodiques, peu à peu compensée par l'augmentation de celle des protéines.

Dans le cas des solutions à base de protéines, la précipitation d'un véritable complexe peu soluble phosphates-protéines-calcium par une interaction des deux premières entités doit maintenant être envisagée sous un angle plus général, notamment par interaction des deux dernières. Le très faible niveau du dépôt à partir des deux protéines essayées $(\alpha \mathrm{lb}$ et $\beta \mathrm{lg}$ ) reste du même ordre de grandeur à la suite de l'addition de calcium, légèrement supérieur toutefois (témoin et cathodique). L'interaction se fait au minimum par liaisons ioniques au niveau des fonctions carboxyliques (acides aspartique et glutamique), mais aussi par complexation, ce qui est conforme aux idées classiques (Weir et Hastings, 1936 ; Greenberg, 1944). Lorsque ces mêmes protéines se trouvent en présence à la fois d'ions calcium et d'ions phosphates, les masses de dépôt sont considérablement modifiées. En l'absence de polarisation, la présence d' $\alpha$-lactalbumine divise par dix l'encrassement par rapport à l'UF. Ceci s'interprète par une complexation $\alpha$-lactalbumine (non dénaturée à $\left.72{ }^{\circ} \mathrm{C}\right)$ - calcium qui atténue le dépôt. Par contre, pour la $\beta$-lactoglobuline, le dépôt est multiplié par deux malgré une possibilité de complexation protéine - calcium en milieu homogène. La précipitation de la $\beta$-lactoglobuline par dénaturation à cette température entraîne un petit excès de phosphate. L'IR permet de préciser que la structure du dépôt est bien, comme pour l'UF, largement majoritaire en phosphates du type apatite. Une interaction des phosphates avec l'ensemble Ca-protéines intervient donc, diminuant la faible solubilité des apatites (espèces essentielles mesurées). Une polarisation cathodique favorise la précipitation des phosphates de calcium : le dépôt en présence d'alb est 25 fois plus important $\left(17 \mu \mathrm{g} \cdot \mathrm{cm}^{-2} \cdot \mathrm{mn}^{-1}\right)$. L'amplitude des écarts observés avec les deux autres systèmes s'atténue normalement (UF : 22 $\mu \mathrm{g} \cdot \mathrm{cm}^{-2} \cdot \mathrm{mn}^{-1} ; \mathrm{UF}+\beta \mathrm{lg}: 26 \mu \mathrm{g} \cdot \mathrm{cm}^{-2} \cdot \mathrm{mn}^{-1}$ ) mais l'ordre croissant des dépôts dans le sens $\alpha$ lb + UF $<$ UF $<\beta l g+$ UF se trouve conservé, ce qui confirme les conclusions précédentes.

Pour les fluides à base de caséinate (sans phosphate), l'augmentation de la masse en polarisation cathodique s'explique par la migration des ions $\mathrm{Ca}^{2+}$ vers l'interface : la force ionique, modifiée localement, favorise la précipitation de la caséine, d'autant plus, d'après BAUMY et BRULÉ (1986), que la concentration en calcium est élevée, à partir d'une certaine concentration critique. L'association du calcium avec la caséine (groupements phosphosérine) est d'ailleurs répertoriée (Stability Constants, 1971). Dans le domaine anodique, les fluides 
contenant des caséines (y compris le lait) donnent les dépôts les plus importants en raison de l'abaissement du $\mathrm{pH}$ à l'électrode. L'influence de la teneur en calcium pour les solutions de caséinate serait toujours liée à la charge superficielle négative de la caséine, d'autant moins importante (et plus aisément compensée par la charge des ions $\mathrm{H}^{+}$) que la teneur en calcium est élevée. FORT (1983) a également expliqué la formation, par une solution de caséinate de sodium, d'un dépôt gélatineux sur une électrode en acier inoxydable polarisée anodiquement, en faisant intervenir la migration et l'acidification de la caséine chargée négativement.

Pour le lait, l'encrassement en anodique augmente de façon considérable alors que, pour le lactosérum, il diminue pour ensuite augmenter légèrement. Dans le cas du lait, la précipitation des phosphates est extrêmement réduite et malgré tout la masse de dépôt augmente. Les résultats obtenus avec le caséinate de sodium, en l'absence de $\mathrm{PO}_{4}$ (et donc sans possibilité de former des phosphates) mais en présence de $\mathrm{Ca}$, montrent que le caséinate donne un encrassement abondant, variable suivant la teneur en Ca mais proche de celui produit par le lait. Il est donc clair que dans le cas du caséinate mais aussi dans celui du lait, ce sont les caséines qui sont responsables de l'accroissement du dépôt. Par contre, sous polarisation cathodique, l'encrassement augmente moins fortement pour le lait que pour le lactosérum, la tendance générale étant cependant la même. On observe d'ailleurs la même augmentation aussi bien pour l'UF que pour les solutions d' $\alpha \mathrm{lb}$ et de $\beta \mathrm{lg}$ en présence de $\mathrm{PO}_{4}$ et $\mathrm{Ca}$, augmentation qui ne peut être due qu'à la précipitation de phosphate de $\mathrm{Ca}$ et à la formation de complexes avec les carboxyles des citrates ou avec les protéines. Le dépôt avec le lait est moindre qu'avec le lactosérum, cette différence étant probablement due à l'inhibition partielle, par la caséine, de la précipitation de phosphate de calcium.

Les modifications de composition des dépôts donnés par les différents fluides montrent donc que l'encrassement par le lactosérum et le lait en conditions naturelles (donc sans courant électrique) s'élabore à partir d'une interaction phosphate de calcium et protéines qui forment un complexe peu soluble emprisonnant également des lipides. Dès les premiers instants, ces différents éléments sont présents et les protéines sont très largement majoritaires. La croissance ultérieure est fondamentalement liée à la présence de $\mathrm{Ca}$ et $\mathrm{PO}_{4}$. En l'absence de $\mathrm{PO}_{4}$, une protéine, même en présence de $\mathrm{Ca}$, ne peut contribuer à la croissance du dépôt.

Les surtensions (mesurées après impulsion de courant pendant $30 \mathrm{~s}$ ) paraissent indépendantes des masses de dépôt, tout au moins dans le domaine anodique, vraisemblablement en raison, aussi, de l'oxydation du titane. Par contre, dans le domaine cathodique, il existe une relation entre l'encrassement et les surtensions mesurées. Ainsi, à l'instant initial, les surtensions sont très voisines pour la solution de « $\mathrm{PO}_{4}+\mathrm{Ca}$ », le lactosérum et l'électrolyte témoin (UF modifié sans $\mathrm{PO}_{4}$ et sans $\mathrm{Ca}$ ), ce qui indique que ces fluides modifient peu la surface. En revanche, dans le cas de l'UF, les surtensions, plus élevées, s'expliquent par la formation d'une couche homogène de dépôt qui isole partiellement le métal de la solution. La similitude entre l'UF modifié et la solution de $4 \mathrm{PO}_{4}+\mathrm{Ca}$ » se retrouve d'ailleurs au niveau de l'allure des courbes où l'existence d'un minimum peut s'expliquer à la fois par la réduction de la couche passive qui n'a pas pu se stabiliser et la réduction de l'eau ou de 
l'oxydant. Dans le cas du lactosérum, l'absence de réduction notable du film d'oxyde s'explique par l'existence d'un film isolant le métal, ce qui n'est pas̄ contradictoire avec le faible niveau de surtension car le dépôt formé est un complexe de phosphate de calcium amorphe et de protéines dont les propriétés conductrices seraient proches de celles du solvant. En présence d'UF (qui ne contient pas de protéines) la nature du dépôt apatites quasicristallines, peut expliquer le niveau élevé de surtension.

Après 20 minutes d'encrassement, les surtensions obtenues avec la solution de " $\mathrm{PO}_{4}+\mathrm{Ca}$ » et l'UF modifié (sans $\mathrm{PO}_{4}$ et sans $\mathrm{Ca}$ ) sont à nouveau très proches mais un peu plus élevées qu'à $t=0$; par contre, celles obtenues avec le lactosérum augmentent considérablement et tendent à se rapprocher de celles de l'UF. Elles révèlent que la solution de " $\mathrm{PO}_{4}+\mathrm{Ca}$ " conduit à un dépôt qui recouvre mal la surface tandis que le lactosérum donne un dépôt dont la proportion de phosphate a augmenté, ce qui modifie fortement la surface du matériau. L'allure des courbes, pour les quatre solutions, devient semblable : elles tendent toutes vers une limite, différente suivant les fluides. Ceci est lié au fait qu'après $20 \mathrm{mn}$ d'encrassement, la réduction de l'eau s'effectue sur la couche d'oxyde stabilisé au contact du fluide et l'on n'observe plus de minimum, quel que soit le fluide.

\section{Conclusion}

L'application d'un courant électrique à du titane ne permet pas de réduire l'encrassement par des fluides tels que le lait ou le lactosérum, sauf dans un domaine de polarisation malheureusement inapplicable aux aciers inoxydables pour des raisons de résistance à la corrosion.

L'ensemble des résultats obtenus permet de mieux comprendre l'influence d'un champ électrique sur l'encrassement des surfaces d'échange de chaleur par des fluides complexes comme le lait et le lactosérum. Ainsi, sous polarisation cathodique, l'élévation locale du $\mathrm{pH}$ favorise la précipitation des phosphates de calcium, mais aussi de la caséine. Sous polarisation anodique, l'abaissement du $\mathrm{pH}$, au contraire, favorise la précipitation des protéines solubles, mais surtout celle de la caséine dans le lait et les solutions de caséinate de sodium; pour ces dernières, elle est d'autant plus aisée que la teneur en calcium est élevée.

Du point de vue du mécanisme de l'encrassement, les résultats confirment que les phosphates, le calcium et les protéines participent conjointement à l'élaboration du dépôt, et que lors de la phase de croissance ces mêmes éléments forment un complexe triple peu soluble impliquant en particulier les groupements carboxylates des acides aspartique et glutamique des protéines solubles ou de la phosphosérine du lait.

Enfin, dans le domaine cathodique, il semble exister une certaine relation entre les surtensions, la nature et peut-être la masse de dépôt. L'analyse des courbes transitoires permet d'obtenir des informations recoupant celles recueillies par l'analyse qualitative et quantitative des dépôts. La comparaison de la réponse électrochimique d'un système encrassant avec celle d'un témoin non encrassant peut donner des informations sur le recouvrement de la surface, 
l'adhérence et la qualité isolante du dépôt. En conséquence, moyennant quelques recherches sur l'optimisation des conditions électrochimiques (courant, durée d'impulsion) et l'étalonnage par rapport à un fluide non encrassant, des mesures de surtensions pourraient servir de principe de base à un capteur d'encrassement.

Reçu le 7 novembre 1986.

Accepté pour publication le 25 mars 1987.

\section{Remerciements}

Nous remercions bien vivement nos collègues Alice PIERRE et J.-J. BAUMY pour la préparation et la fourniture des protéines purifiées ( $\alpha$-lactalbumine, $\beta$-lactoglobuline) utilisées dans ce travail ainsi que G. BRULÉ pour sa contribution précieuse au choix des solutions modèles et à la discussion des aspects biochimiques.

Les conseils de F. CEEURET (Jeune Equipe "Génie des procédés de séparation ». C.N.R.S Rennes) ont contribué à résoudre certaines difficultés d'ordre électrochimique rencontrées sur la maquette.

\section{Références bibliographiques}

Bard S., Faulkner R., 1983. Electrochimie. Ed. Masson, Paris.

Baumy J.-J., Brulé G., 1986. Etude comparée de la solubilité et de la viscosité des solutions de caséinate et de paracaséinate de sodium en présence de calcium. Lait, 66, 65-77.

Beljelti S., Ben Bachir A., Dumas J.-P., El Adoui S., Jounnneau A., Petit M.-C., 1980. Etude des transformations de quelques protéines au contact du nickel. Mater. Chem. (5), 718.

Daufin G., Kerhervé F.-L., Quemerais A., 1985. Electrochemical potential influence on stainless steel or titanium heat exchange surface fouling by sweet whey. In : Fouling and Cleaning in Food processing. Ed. Lund D., Plett E., SANDU C., USA, University of Madison Wisconsin, 263-275.

Daufin G., Labbé J.-P., Quemerais A., Brulé G., Michel F., Roignant M., Priol M., 1987. Analytical study of a heat exchange surface by whey, milk and model fluids. Lait, 67 (3), 339364.

Digby D., Mac Donald, 1977. Transient techniques in electrochemistry. Ed. Plenum Press New York.

FORT J., 1983. Contribution à l'étude des interactions caséinate de sodium - acier inoxydable austénitique ou argent; mise au point et développement de films adsorbés à la surface des matériaux. Thèse Docteur-Ingénieur, Toulouse.

Grasshoff A., 1985. Zum Einfluss des Potentialfeldes auf die Belagbildung im Plattenherhitzer. Int. Z. Lebensm. Technol. Verfahrenstech., 36 (1), 6, 8-9.

Greenberg D.M., 1944. Interaction between the alkali earth cations, especially calcium and proteins. Adv. Prot. Chem., 1, 121-151.

Ivarsson B., JonsSON S., LundSTROM I., 1981. Ellipsometrical and electrochemical studies of adsorption at solid/liquid interface. In : Fundamentals and applications of surface phenomena associated with fouling and cleaning in food industry. Ed. HALLSTROM B., LUND D.B., Tragardh C., Sweden, Lund University, 269-278. 
Landolt D., 1984. Allgemeine Uberlegungen Zum Einfluss der Stromform auf die Metallabscheindung. Oberflache/Surf., 2, 5-6.

Le Duigou A., 1982. Contribution à l'étude des mécanismes de l'entartrage des appareils sanitaires électriques. Thèse : ENSAM, Paris.

Legeros R.Z., Trautz O.R., Legeros J.P., Klein E., 1967. Apatites crystallites : effects of carbonate on morphology. Sciences, 155, 1409-1411.

Pierre A., Fauquant J., 1986. Principes pour un procédé industriel de fractionnement des protéines du lactosérum. Lait, 66, 405-419.

Roignant M., Daufin G., Michel F., 1983. Dispositif expérimental pour étudier l'encrassement des échangeurs de chaleur alimentaires. Lait, 63, 363-390.

Stability constants, 1971. Supplément $\mathrm{n}^{\circ}$ 1, Special Publication 2. The Chemical Society, London.

Tai A., Messin G., Pagetri J., 1985. Electrodéposition d'alliages étain-plomb : étude des courbes transitoires. Galvano. Organo. Trait. Surf., 558.

Weir E.G., Hastings A.B., 1936. The ionization constants of calcium proteinate determined by the solubility of calcium carbonate. J. Biol. Chem., 114, 397-406. 